\title{
A Neutron Detector Based on Microchannel Plates
}

\author{
D. W. MacArthur
}

\section{DISCLAIMER}

This report was prepared as an account of work sponsored by an agency of the United States Government. Neither the United States Government nor any agency thereof, nor any of their employees, makes any warranty, express or implied, or assumes any legal liability or responsibility for the accuracy, completeness, or usefulness of any information, apparatus, product, or process disclused, or represents that its use would not infringe pri.ately owned rights. Reference herein to any specific commercial product, process, or service by trade name, trademark, manufacturet. or otherwise does not necessarily constitute or impl; its endorsement, recommendation, or favoring by the United States Guvernment or any agency thereof. The views and opinions of authors expressed herein do not necessarily state or renlect those of the United States Government or any agency thereof. 
T. ABLE OF CONTENTS

Page

INTRODUCTION

CONCEPT 1

MASS, ELECTRONICS, AND COST 23

$\begin{array}{ll}\text { MCP DESCRIPTION } & 4\end{array}$

MCP CHARACTERISTICS $\quad 4$

$\begin{array}{ll}\text { CONCLUSIONS } & 5\end{array}$

$\begin{array}{lr}\text { REFERENCES } & 6\end{array}$ 


\title{
A NEUTRON DETECTOR BASED ON MICROCHANNEL PLATES
}

by

\author{
D. W. MacArthur
}

\begin{abstract}
We propose a large-area neutron detector design based on microchannel plates (MCPs). Two characteristics of the MCP make it ideal as a high-rate neutron decector: (1) its signals can have a very fast rise time, and (2) it can count at a high rate. The MCP-based detector could use both the high-voltage power supplies and the readout electronics designed for a neutron detector based on the multiwire proportional chamber (MWPC).
\end{abstract}

\section{INTRODUCTION}

In this paper we describe a neutron detector using microchannel plates (MCPs) as its active elements. The MCP-based detector arriuy is designed to respond to a few high-energy neutrons in the presence of a very large number of gamma rays and low-energy neutrons. Unfortunately, an MCP is intrinsically sensitive to gamma rays, a fact which we hope can be overcome by its high counting rate capability. The detector is being developed jointly with Ball Aerospace Systems Division, where all necessary fabrication would be done. Conceptually, the MCP-based detector is very similar to the detector based on the multiwire proportional chamber (MWPC) ${ }^{1}$ and uses much of the same associated electronics. The two detectors are identical in design. except that the MWPC layers are reolaced with MCP layers. Therefore, the MWPC design paper $^{1}$ should be read in conjunction with this one, which only touches on the differences between the two designs. The MCP-based design is not com- pletely defined, so some values are still undetermined at this point.

\section{CONCEPT}

Although all actual detectors will contain more layers, Fig. 1 illustrates three layers of the MCPbased geutron detector. Each layer consists of a polyethylene sheet (4) to generate protons from incident neutrons; a combination high-voltage electrode and range filter (2); a layer of MCPs (3a) to detect the proinns; and a foil anode ( $3 b$ ) to collect the charge generated by the MCPs. In the front of the stack is a sheet of polyethylene (1) which acts both as a protective cover and the first converter. In back of the stack is another polyethylene sheet (5) which acts as a cover only. Comparing Fig. 1 with Fig. 2 (which shows a schematic of three segments of the MWPC-based design) further illustrates the design similarities between the two detectors. In these figures, the corresponding layers $1,2,4$, and 5

* Ball Aerospace Systems Division, P. O. Box 1062, Boulder, CO 80306. 
have identical functions in both detectors. Only the actual active detectors, layer 3 , are different. In the MWPC design, layer 3 is a multiwire chamber; while in the MCP design, layer 3 is a inicrochannel plate and a readout anode.

Figure 3 details the proposed construction method for the layers of the MCP detector. Microchannel plates are iaminated onto a poly-

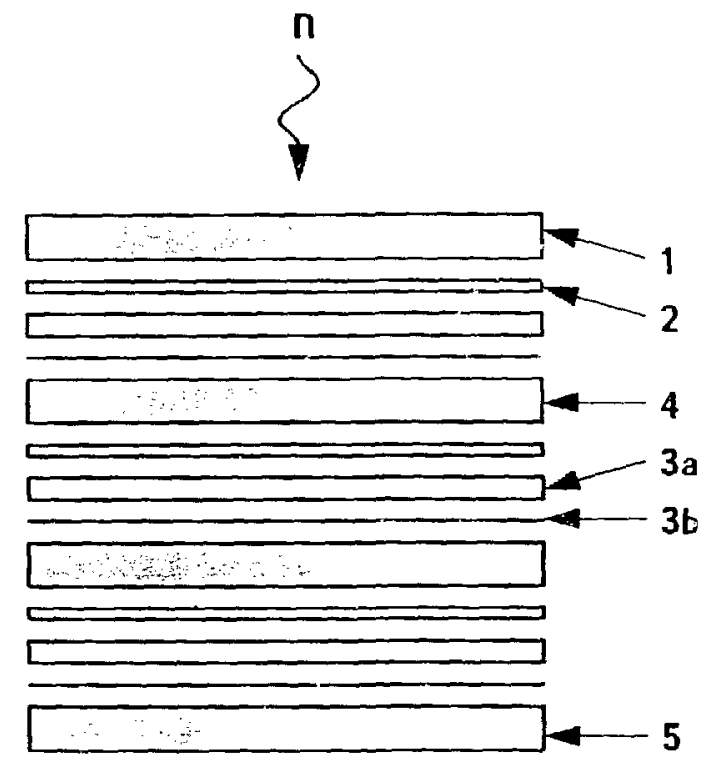

Fig. 1. Three layers of an MCP-based detector stack Layers 1, 4, and 5 are polyethylene; number 2 layers are aluminum range filters/high-voltage elecirodes; and number 3 layers are the MCP arrays, which are made up of MCPs (3a) and another electrode (3b). ethylene converter that already has the anode readouts and the range filter/high-voitage electrode deposited on it. The negative high voltage (about $2000 \mathrm{~V}$ ) applied to the electrode is to bias the subsequent MCP layer. The anodes are near ground potential so that there is a reverse bias across the polyethylene. However, this bias is relatively small (compared to $\mathrm{MeV}$ particle energies) and not ex. pected to be a problem.

Although each detector module has two laycrs of aluminum (the range filter and readout anode), the readout anode layer is expected to be very thin compared with the filter layer, so its filtering action can be ignored. Many of these sheets are stacked to form a single neutron detector. Clearly the front and back layers would have to be somewhat different from the rest.

Conceptually, any size array of MCPs is possible. The first prototype will be a single MCP across (one pixel) and ten layers thick. Each MCP will have a single readout anode and a single highvoltage connection. Thus, the prototype will be a cylinder $25 \mathrm{~mm}$ in diameter and about $3 \mathrm{~mm}$ long with 20 electrical connections. In advanced versions all of the plates may operate from the same highvoltage supply, but for the prototype we will want the flexibility of separate supplies. Figure 4 shows a moderate-sized (10 × 10) MCP array, which is composed of the signal connector, $100 \mathrm{MCPs}$ (assumed iound with a $25-\mathrm{mm}$ diameter), and the polyethylene mounting sheet/converter. The readout anodes are hidden behind the individual MCPs. Each anode is connected to the readout connector by a trace on the polyethylene. The range filter/high-voltage electrode is hidden behind the polyethylene sheet. For structural reasons, larger

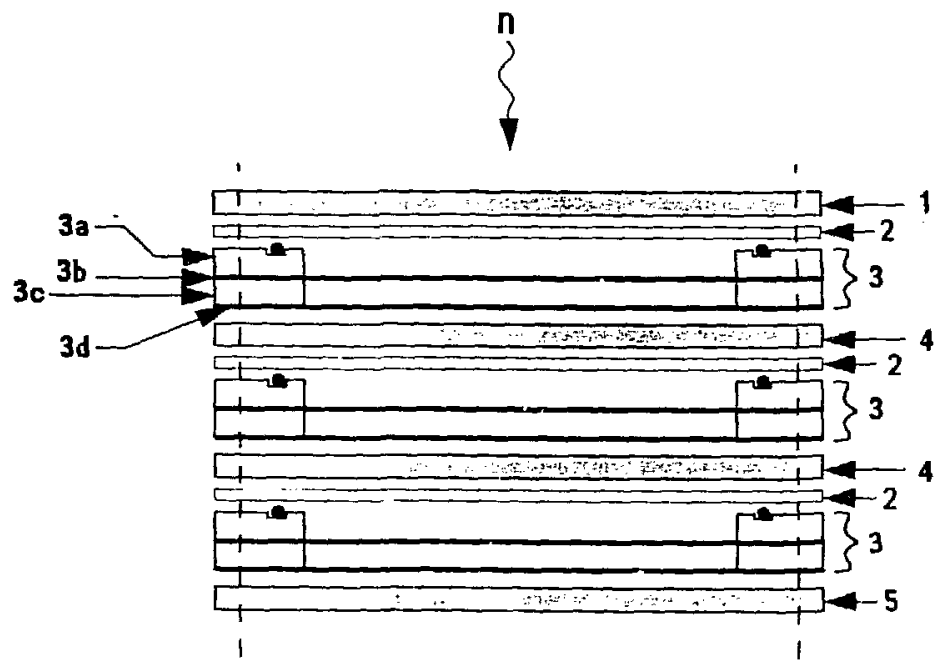

Fig. 2. Three segments of an MWPCbased detector stack for comparison with Fig. 1. Polyethylene $(1,4,5)$ and aluminum (2) layers are the same as Fig. 1. In this case, the actual detectors (3) are MWPCs made up of aluminum frames $(3 a, 3 c)$, wires $(3 b)$, and aluminized mylar windows. 
Fig. 3. Cross-sectionai view of a laminated MCP/range filter/polyeihylene convere: composite. The readout anode is a thin electrode used to bias the following $M C P$ and readout its data.

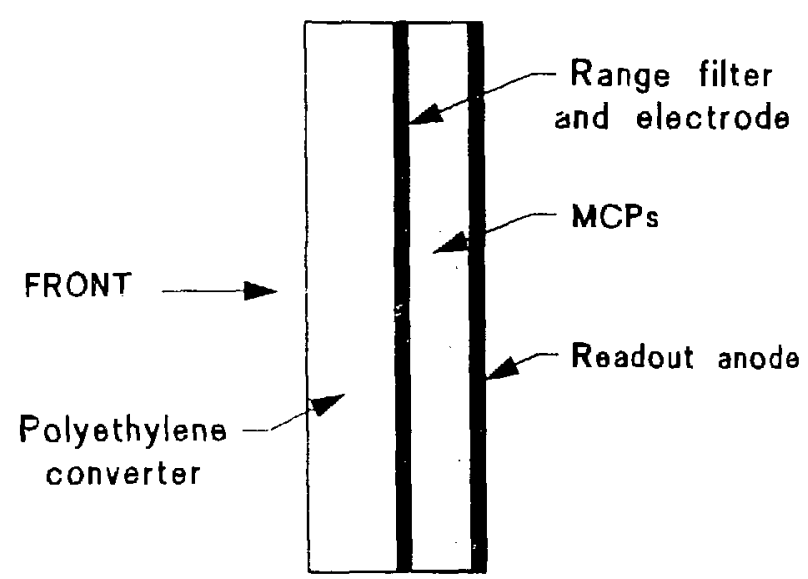

MASS, ELECTRONICS, AND COST

Because the thickness of the MCP detector will be based on the range of several MeV protons (the same as the MWPC-based detector), their mass is expected to be similar. The mass of the MCP-based detector's support system should be less than for MWPCs because no gas handling system, gas storage tanks, or pressure chamber will be required. More detailed estimates of its mass will be made as the design progresses.

The electronics used to solve data readout problems in the MWPC-based detector ${ }^{1}$ can be used in the MCP-based detector because similar problems exist. The high-voltage power supplies specified for the MWPCs are also directly applicable to the MCPs. The outputs of the $10 \times 10$ array of microchannel plates will be made pin-forpin compatible with the outputs of the 10-in. MWPC. As a result, the readout electronics package can fit either detector.

Standard 25-mm diameter microchannel plates vary enormously in cost. Selected curved channel plates used by Ball Aerospace cost about $\$ 5,000$ in single quantities while the plates used by the military in night-vision goggles cost about $\$ 200$ in large quantities. ${ }^{2}$ Low-grade plates may be acceptable for our design; and because our requirements will be large, the price nay be near the low end. A plate manufacturer, Galileo Electro-Optics Corporation, ${ }^{*}$ is being queried on this point. Even at $\$ 2.00$ per plate, this technology may be too expensive for a large detector. detectors may be made up of many $10 \times 10$ arrays mounted side-by-side.
Fig. 4. MCPs in a $10 \times 10$ array mounted on $a$ polyethylene backing sheet. All signals are brought out to the signal connector.

* Galileo Electro-Optics Corp., Galilar, Park, Sturbridge, MA 01518. 


\section{MCP DESCRIPTION}

J. G. Timothy ${ }^{3}$ has written an excellent description of MCP operation, so the present discussion will be brief. An MCP can be thought of as an array of continuous photo-multiplier tubes. Figure 5 illustrates a cutaway view of a single channe!. The inside surface of the hollow channel is coated with a semiconducting material, which is also a secondary electron emitter. This semiconducting inner surface insures that there is a uniform electric field inside when high voltage is applied to the electrodes at either end of the channel. The primary radiation (in this case protons) strikes the inner surface of the channel, creating several secondary electrons. These electrons are then accelerated down the channel until they hit the wall again and knock out even more electrons. This electron multiplication continues down the channel until a large electron cloud exits from the far end. The resulting charge can be collected on a conducting anode, and the induced current pulse amplified in a conventional manner.

A single channel is known as a channel electron muliplier (CEM). Many CEMs can be mounted side-by-side to form an array (Fig. 6) called a microchannel plate (MCP). The channels are called "micro" because each one is only about 12 microns in diameter. This diameter and other specifications mentioned in Table I are typical values derived from Ref. 4. It should be emphasized that these values are typical and are not necessarily representative of any specific microchannel plate. In contrast to the more common straight channel MCPs, this design uses curved channel MCPs as shown in Fig. 7.

\section{MCP CHARACTERISTICS}

The MCP has many advantages as a high-rate particle detector. We have listed some of them, along with a few disadvantages, below. (Many of the results mentioned below were obtained around 1980 and may be somewhat dated.)

\section{Advantages}

1. Signals from an MCP exhibit a very fast rise time. Wiza ${ }^{4}$ reports rise times of $<500 \mathrm{ps}$, which is advantageous in separating signals in a high-rate counting environment.

2. MCPs are high-gin devices $\left(10^{5}\right.$ to $\left.10^{7}\right)$ allowing detection of single particles. ${ }^{3,4}$

3. A maximum count rate capability of $10^{7}$ counts $\mathrm{cm}^{-2} \mathrm{~s}^{-1}$ was reported in $1981^{3}$ It has been reported that $10^{8}$ is reachable today, and we feel that another order of magnitude improvement may be püssible using specially designed MCPs. ${ }^{2}$

4. The expected lifetime of an MCP is long. (Timothy ${ }^{3}$ reported an active lifetime of $2.5 \times 10^{33}$ counts $\mathrm{cm}^{-2}$ )

5. In a good vacuum ( $<10^{-6}$ torr), an MCP exhibits a low dark count rate $\left(<1 \mathrm{~Hz} / \mathrm{cm}^{2}\right){ }^{4}$ This performance can be achieved without any cooling.

6. Large doses of gamma radiation do not adversely affect MCP operation. Timothy ${ }^{3}$ found that a dose of $10^{6}$ rads of $1-\mathrm{MeV}$ gamma radiation did

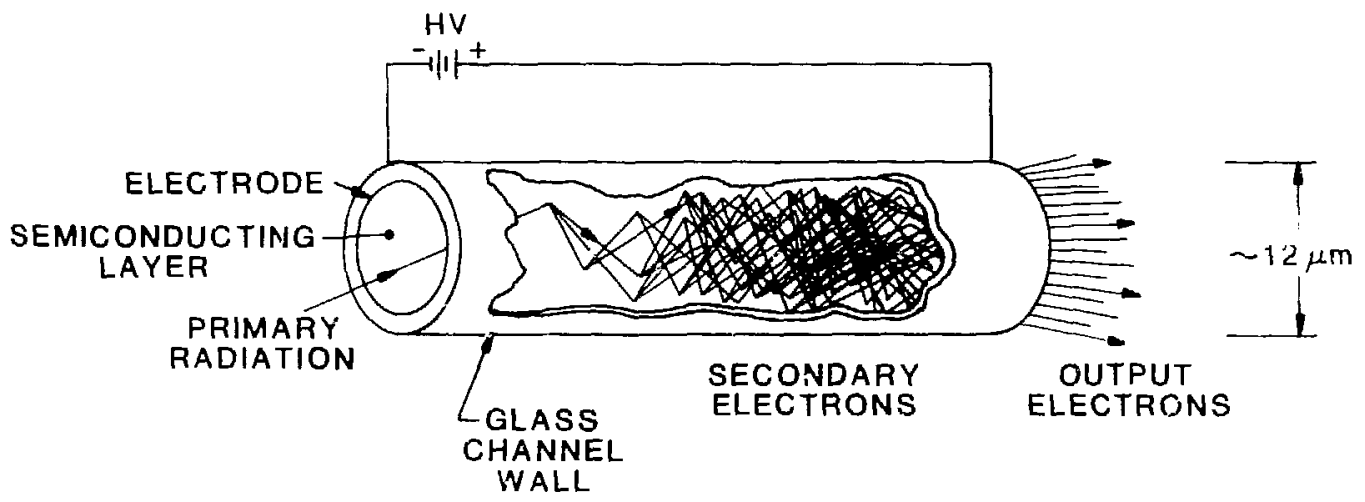

Fig. 5. Cutawery view of a single straight MCP rube. 


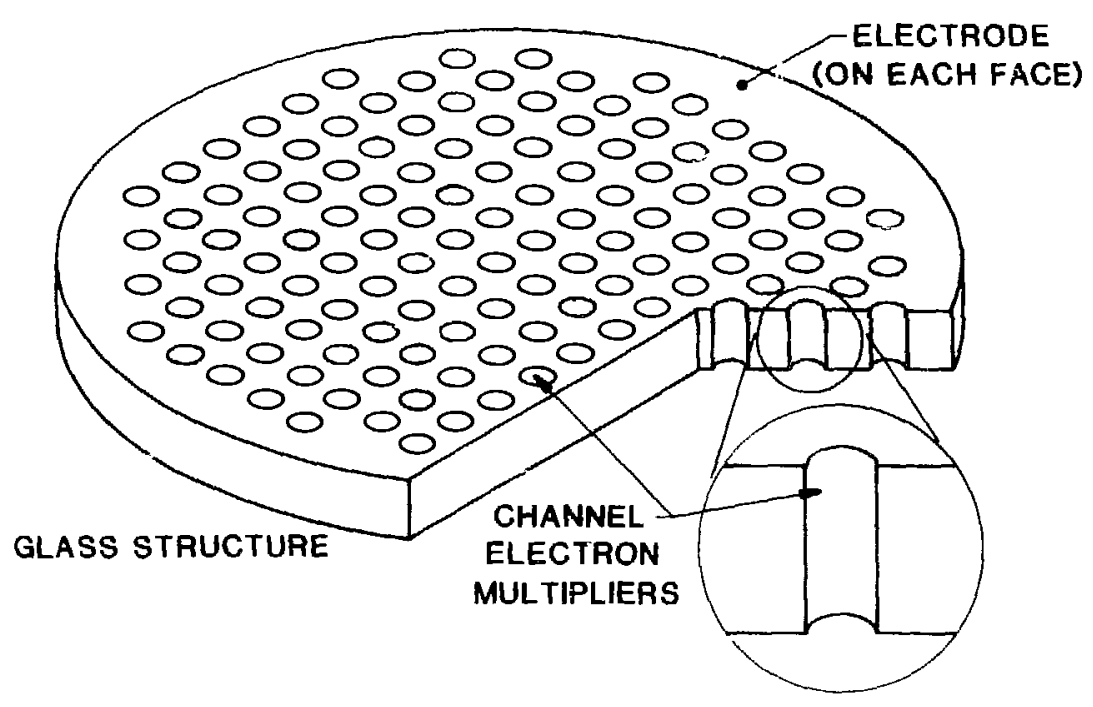

Fig. 6. Cutaway view of an entire straight channel MLP.

\section{TABLE I}

\section{TYPICAL MCP SPECIFICATIONS}

\section{MCP Parameter}

Channel diameter

Channel spacing

Channels per plate

Plate diameter

Plate thickness

\section{Value}

$12 \mu$

$15 \mu$

$1,000,000$

$2.5 \mathrm{~mm}$

$0.1 \mathrm{~mm}$ not degrade an MCP's response. Many other detector components will be damaged before the MCP.

7. An MCP consists of a single piece of ceramic, thus, it is well able to withstand mechanical shocks. The entire sensor structure will have no moving or loosely attached parts.

8. An intrinsically rugged device, the MCP is operable in magnetic fields of up to $1 \mathrm{kG}$ and can withstand radiation, vacuum, and tcmperature extremes.

9. The MCP has very small independent channels, each of which operates as an individual detector, translating into a very small pixel size.
10. The electronic pixels of an MCP :aray are more square than linear, making them much easier to interpret by an electronic trackfinder. This simplifies the readout electronics for the MCPbased detector.

\section{Disadvantares}

1. The MCP, being ceraric, has a fairly high mass and density.

2. Along with many other types of detectors, the MCP requires a moderately high operating voltage (about $2000 \mathrm{~V}$ ).

3. The MCP is basically a saturated gain device, therefore the output pulse will have the same magnitude no matter how much energy is deposited in the channel. Thus, only coincidence between MCP layers can be used to discriminate against unwanted radiations. (Because the output depends slightly on the deposited cnergy, some particle discrimination may be possible. ${ }^{2}$ ) However, gain saturation does mean that the individual outputs are practically digital, which may simplify the readout electronics.

\section{CONCLUSIONS}

Ball Aerospace is considering a proposal to build the single-pixel version of this detector. This prototype would have 10 layers of microchannel 


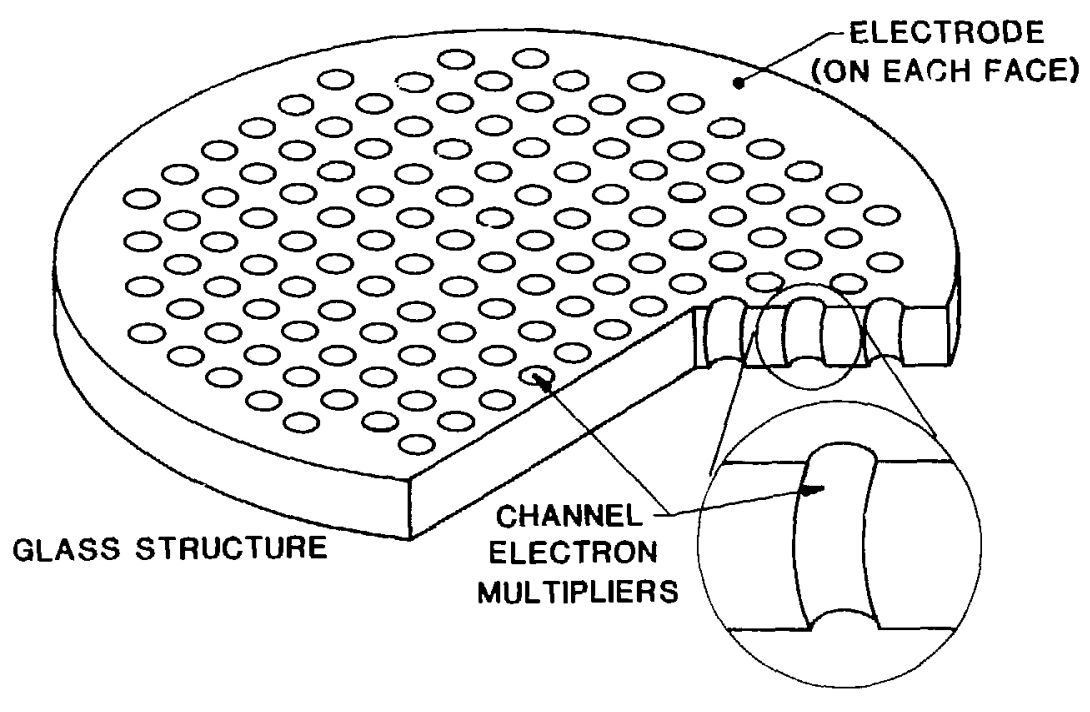

Fig. 7. Culaway view of a curved channel MCP.

plates. If the prototype performs as expected, we would build a somewhat larger version (perhaps 10 layers of $10 \times 10 \mathrm{MCPs}$ ). This medium-sized detector will help evaluate many problems associated with building a much larger detector. We expect that our association with Ball Aerospace would speed up the development of this concept considerably.

The MCP appears to have many advantages as the basis for a large-area neutron detector. To the best of our knowledge, MCPs have not been used in this configuration before, so a number of physics questions need to be answered before further progress can be made.

\section{REFERENCES}

1. D. W. MacArthur, "Design Report on a 10-in. Multiwire Proportional Chamber and Associated Electronics: May 1986," Los Alamos National Laboratory report LA-10916-MS (August 1986).

2. R. L. Bybee, private communication.

3. J. G. Timothy, Rev. Sci. Instrum. $\underline{52} 1131$ (1981).

4. J. L. Wiza, Nucl. Instrum. Methods 162 587-601 (1979).

5. J. G. Timothy, G. H. Mount, and R. L. Bybee, "Multi-anode Microchannel Arrays," IEEE Transactions on Nuclear Science NS-28 (1) (1981). 